\title{
Interactive comment on "Climatic subdivision of Heinrich Stadial 1 based on centennial-scale paleoenvironmental changes observed in the western Mediterranean area" by Jon Camuera et al.
}

\section{Anonymous Referee \#3}

Received and published: 6 February 2020

In this manuscript, Dr Jon Camuera and co-authors propose to divide the Heinrich Stadial 1 (HS1), one of the coldest and driest phases of the last glacial, on the basis of the changes in moisture availability and temperature inferred from the high-resolution pollen sequence of the Padul wetland (southern Spain). The authors also use sedimentological data to support their argumentation. The chronological framework is based on a number of radiocarbon dates from bulk sediment and classical age-depth modelling. The chronology of their record led the authors to suggest the HS1 in the western 
cores and most (if not all) of the European well-dated sedimentary records currently available. The research questions addressed are timely and of great relevance for the palaeoclimatic community, the methods used are mostly appropriate, and I acknowledge the effort the authors have made to discuss their results in the context of previously published regional palaeoclimatic evidence. However, I have noticed several important issues that the authors should address to make their arguments more convincing. At the moment, after reading carefully the manuscript, I am afraid that the main conclusions of the research are not fully supported by the data presented. In the following paragraphs, I will elaborate on why I got this impression.

\section{GENERAL COMMENTS}

1. A high number of radiocarbon dates does not necessarily mean that the chronology is robust. In this case, as shown in Figure 2 and Table S2, the number of radiocarbon dates available is truly impressive but all the accepted ones are on organic bulk sediment and bulk carbonate. The risk of hard-water effect is notable when dating bulk sediment from lakes whose catchments are mostly on calcareous bedrock. This holds particularly true for sections of the sedimentary sequence with higher shares of inorganic matter. The bedrock in the Padul-Niguelas basin is mostly calcareous (see Ramos-Roman et al., 2018), which is relevant also for this work and should be indicated in the main text or at least in the SI. This would contribute to explain the offset of ca. 1000 years observed in the Padul record at the beginning and the end of the HS1 compared with most of the well-dated records from Greenland and elsewhere in Europe. To have a sound support for their conclusions in this regard, the authors should provide a chronology based on short-lived terrestrial plant macrofossils or try to find out what the reservoir age for this period is. In any case, I consider that it is important that the authors discuss the limitations of their chronology.

2. Although I also see some patterns in the pollen data and the synthetic pollen-based indices suggestive of changes in the local climate, the record is quite "noisy". I was wondering whether the authors have tried to check and validate their visual delimi- 
tation of the phases and sub-phases within the HS1 using numerical tools such as zonation. Divisive (e.g. optimal splitting) or agglomerative (e.g. CONISS) methods to delimit groups of samples with similar pollen assemblages in an objective manner (pollen zones) would be quite appropriate in this case because otherwise the position of the boundaries for the different periods seems to be somehow arbitrary.

3. I acknowledge the interest of using indices to summarize pollen data to facilitate their interpretation, but it is necessary to see the raw data (in the SI if not in the main text) because the abundances of specific taxa may be relevant for the palaeoclimatic interpretation of the results. I would kindly ask the authors to supply a pollen diagram for the relevant period under investigation so the readers can fully assess the significance of the dataset.

4. According to the authors, the HS1 lasted $2.8 \mathrm{kyr}$ in Padul, which is also in agreement with other continental, marine and ice records. Then, do periodicities of 2000 or even 800 years make sense at all? To me, on due respect, perhaps not too much...

\section{SPECIFIC COMMENTS}

Several words are repeated in the title and the keywords, i.e. Climate, Heinrich Stadial 1 and western Mediterranean. Perhaps the authors might consider adding some different keywords to make their paper easy to be found in scientific databases, e.g. southern Iberia, palaeoclimate or pollen analysis.

L18 Replace "generating" with "characterized by"?

L39-40 The authors might consider adding here a very relevant reference about last glacial rapid climatic variability in the North Atlantic context: Sanchez Goni et al. (2008) Quat. Sci. Rev. 27, 1136-1151.

L46 Replace "focus" with "have focused"?

L47 Replace "short-scale" with "short-term"? I think it would be more adequate... 
L49-53 At least part of the content of the SI should be moved here so the reader gets a better idea of the background that justifies this research.

L54 "Sedimentological" instead of "sedimentation"?

L58 There is a mismatch between the time resolution indicated here, i.e. "61-yr", and the values shown in the SI, i.e. "77-yr" and "131-yr". Please, check and be consistent.

Interactive

comment

L81-83 Low percentages of xerophytic pollen during the LGM are quite unexpected. I have been checking the pollen diagram in Camuera et al. (2019) Quat. Sci. Rev. and pines were particularly abundant by that time around Padul. I was wondering whether the authors could provide a plausible explanation for these values because I consider this point would be worth to be discussed.

As I already said in my general comments, I see the patterns that the authors point out but it is also true that there is a significant overlap in the values, especially around the boundaries. The authors could seek statistical support for their inferences using a zonation procedure. HS1a.1-HS1a.3 and HS1c.1-HS1c.3 are hard to accept. Perhaps increasing the number of samples would bring support to this proposal, but with the current record it is not possible to assess whether peaks in a single sample are palaeoclimatically meaningful or just outliers.

L129-153 The authors should discuss here the potential hard-water effect in their chronology.

L146-148 References supporting this statement are needed.

L150 "Conditions" could be deleted.

L159 How the authors would explain the ca. 1000-yr offset between the onset of the Lateglacial Interstadial at Padul and most well-dated European records, including those from southern Europe, e.g. Monticchio, Trifoglietti?

Printer-friendly version

SUPPLEMENTARY INFORMATION

Discussion paper 
L21 Please delete "one of", because it is certainly the most recent HS.

Regional and local settings. It is highly relevant that the authors inform about the CPD bedrock in the catchment.

Chronology. Please, indicate which dates are new and which were published in previous studies.

Interactive comment

L93 For the future, it would probably be worth to discuss the presence of Carpinus.

Spectral analysis and filtering. At least part of this text should be moved to the main text.

Interactive comment on Clim. Past Discuss., https://doi.org/10.5194/cp-2019-130, 2019. 